\title{
La comprensión de conceptos fundamentales del cálculo mediante Desmos. Una intervención
}

\author{
Understanding Fundamental Concepts of Calculus Through Desmos. An \\ Intervention
}

\section{A compreensão de conceitos fundamentais de cálculo usando Desmos. Uma \\ intervenção}

Erick Radaí Rojas Maldonado

Universidad Michoacana de San Nicolás de Hidalgo, México

errojas@umich.mx https://orcid.org/0000-0003-2521-5107

\section{Resumen}

En este artículo se realiza el diseño de una estrategia metodológica para la comprensión del concepto de derivada. Esto a través de Desmos y el programa "trae tu propio dispositivo" (BYOD, por sus siglas en inglés) en un primer curso de cálculo diferencial a nivel licenciatura, así como mediante la herramienta metodológica heurística de investigaciónacción. Para lograr el objetivo se involucra, además, el método de derivación por incrementos, el cual se codifica para ser incorporado a la plataforma Desmos y con ello realizar animaciones. Posteriormente, se realizan quizzes a través de Kahoot! para evaluar su comprensión. Se concluye que Desmos es un auxiliar de utilidad en la comprensión de conceptos fundamentales del cálculo, ya que permite el esbozo de funciones de manera sencilla; así, da sentido a la matematización, aunque no al cálculo de resultados.

Palabras clave: aprendizaje, derivada, interpretación, límite. 


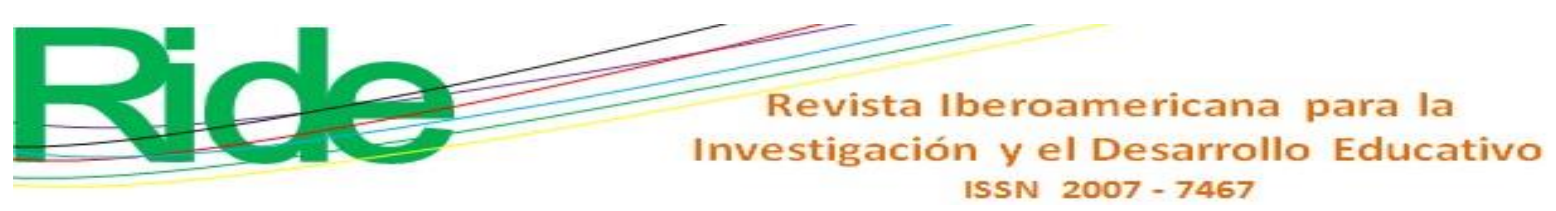

\section{Abstract}

The design of a methodological strategy for the understanding of the concept of derivative is carried out in this article. This through the Desmos software and the bring your own device (BYOD) program in a first course of differential calculus at the degree level, using the action research methodology. Also, it is involved the method of derivation by increments, which is coded to be incorporated into the Desmos platform and thus make animations. Quizzes are then performed through Kahoot! to evaluate the understanding of the students. It is concluded that Desmos is an useful aid in the understanding of fundamental concepts of calculus, since it allows the sketching of functions in a simple way giving meaning to mathematization, but not the calculation of results.

Keywords: learning, derivative, interpretation, limit.

\section{Resumo}

Neste artigo, é realizado o desenho de uma estratégia metodológica para a compreensão do conceito de derivada. Isso através do Desmos e da prática "traga seu próprio dispositivo" (BYOD) em um primeiro curso de cálculo diferencial no nível de graduação, bem como através da ferramenta metodológica heurística da pesquisa-ação. Para atingir o objetivo, também está envolvido o método de derivação incremental, que é codificado para ser incorporado à plataforma Desmos e, assim, fazer animações. Posteriormente, os testes são realizados através do Kahoot! para avaliar sua compreensão. Conclui-se que Desmos é uma ajuda útil na compreensão de conceitos fundamentais de cálculo, pois permite o esboço de funções de maneira simples; Assim, dá sentido à matematização, embora não ao cálculo dos resultados.

Palavras-chave: aprendizagem, derivada, interpretação, limite.

Fecha Recepción: Enero 2020

Fecha Aceptación: Mayo 2020 


\section{Introducción}

Cuando se habla de las dificultades de impartir cálculo diferencial e integral, se habla, en parte, de vencer la mecanización de procesos algebraicos. El límite y la derivada son conceptos primordiales que el alumno debe de comprender para desarrollar la habilidad de calcular e interpretar y, del mismo modo, para incorporar conocimiento posterior dentro de ciencias como la física y las matemáticas. El aprender matemáticas no solo requiere de la habilidad para realizar ejercicios, sino también de plasmar ideas y representaciones a través de símbolos, los cuales representan un movimiento, un estado, una variación o una incógnita. A esa idea plasmada en la forma de una ecuación u operación, si no se conoce su significado, es imposible añadirle mayor conocimiento, lo cual incide en la resolución de un problema. Como lo señalan White y Mitchelmore (1996), la resolución de problemas de aplicación debe acompañarse de un conocimiento conceptual y no de una "comprensión instrumental".

En una investigación relacionada con el proceso/objeto para el caso del límite, Cottrill et al. (1996) subrayan que la dificultad de comprender el concepto de límite radica en que para ello se requiere la reconstrucción de dos procesos coordinados. Más tarde, Sierra, González y López (2000) señalan la dificultad de la compresión de los conceptos de límite y continuidad incluso después del proceso de enseñanza. Mientras que Ferrini-Mundy y Graham (1994) documentaron la dificultad del estudiante de conectar la representación simbólica de una derivada con cualquier tipo de comprensión geométrica. Y una investigación posterior, de Habre y Abboud (2006), señala que, a partir de una enseñanza con énfasis en la visualización, los alumnos entienden a la derivada como un cambio instantáneo o como la pendiente de una curva en un punto. Martínez, López, Gras y Torregrosa (2002), por su parte, describen las aportaciones e insuficiencias de las concepciones históricas de Leibnitz y Cauchy.

En esa línea trazada por los ejemplos arriba citados, en esta investigación se ha comprobado la escasa interpretación de conceptos fundamentales en el cálculo y una habilidad insuficiente en el manejo algebraico en el cálculo de límites e integrales. FerriniMundy y Gaudard (1992) también muestran que una enseñanza basada en procesos algorítmicos es inadecuada para lograr la interpretación correcta de conceptos dentro del cálculo diferencial. Asimismo, Rojas (2015) documenta secuencias didácticas que buscan favorecer la comprensión del concepto de límite de una función, las cuales descansan sobre 


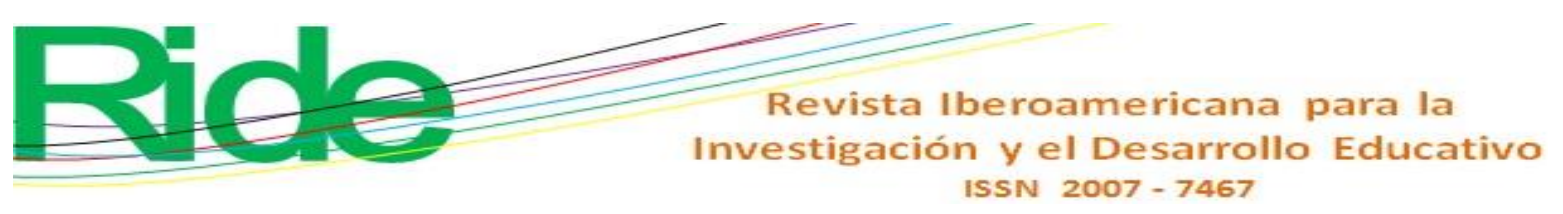

una adecuada comprensión del concepto, lo cual no era así a principios del siglo XIX con Cauchy.

Con base en ello y sabiendo de la importancia del manejo algebraico y la resolución de límites y derivadas, se hace una propuesta a través del dibujo y la animación digital para buscar incidir en la comprensión del concepto de derivada con ayuda de Desmos, una calculadora gráfica de libre acceso y disponible para varios sistemas operativos y dispositivos.

En Rojas (2019), Thomas (2015), Montijo (2017) y Almarshedi, Wanick, Wills y Ranchhod (2017) se señala la importancia del dibujo a través de la calculadora gráfica Desmos y de la transición actitudinal del alumno para aprender a través de la gamificación. Sin embargo, el dibujo por sí mismo no incide en una respuesta, sino más bien en un orden de ideas o pasos para desarrollar, o bien, para plasmar un fenómeno ya sea físico o químico, por mencionar un par de ejemplos.

Sin embargo, el hecho de derivar una función no implica el entendimiento del uso de esta; cuando se habla de derivada se habla de cambios, de variaciones, y quizá no es de interés saber los resultados de esos cambios, sino cómo se originaron y a lo que conllevan; quizá acontecieron en un lapso muy pequeño, lo cual puede pasar desapercibido para el "ojo humano". Así que es necesario redefinir el fenómeno y acotarlo en un cierto intervalo. No obstante, la derivación por incrementos nos permite definir formalmente a la derivada. A este método también se le conoce como la regla de los cuatro pasos, la cual tiene la particularidad de que es necesario involucrar límites; además, permite interiorizar el aprendizaje a diferencia de la derivación por fórmulas; o bien, como diferencial, que provino de un proceso “tormentoso" para Newton y Leibnitz y que vio la luz con la llegada de Cauchy.

El incremento de cualquier función continua obedece a la definición formal de infinitesimal, no tiene sentido utilizar el término diferencial para referirse al incremento (infinitesimal) de una función. Si a esto se añade la sospecha acumulada a lo largo de los años sobre la diferencial y los infinitesimales de servir de base a tratamientos matemáticos poco rigurosos, el terreno resultaba claramente abonado para que la diferencial quedase relegada a un papel marginal en el nuevo marco teórico del cálculo (Martínez et al., 2002, p. 275).

Una vez que el cimiento de la derivada se encuentra fuerte, es posible evolucionar a la instrucción del cálculo de esta a través de procesos algorítmicos. 


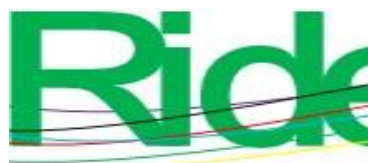

Revista Iberoamericana para la
Investigación y el Desarrollo Educativo
ISSN $2007-7467$

La importancia de conocer la derivación por incrementos radica en la matematización y modelación matemática (Rojas, 2018; Martínez, Cobos y Torres, 2015; Arrieta y Díaz, 2015) para la apropiación del lenguaje y de la síntesis de una conceptualización ya posterior. Además, la recuperación de saberes a priori es importante para crear un andamiaje matemático.

Todo lo anterior constituye un cúmulo de motivos suficientes para realizar una investigación donde se programe con ayuda de la calculadora gráfica Desmos el método de derivación por incrementos y analizar su contribución en la comprensión de la derivada en un curso de cálculo.

\section{Problema de investigación}

¿De qué manera el empleo de la calculadora gráfica Desmos contribuye a comprender la derivada?

\section{Objetivo}

Programar a través de la calculadora gráfica Desmos el método de derivación por incrementos

\section{Método}

El presente trabajo está moldeado por la forma de investigación-acción, pues la intervención y el análisis de esta es lo que nos hace mejorar la comprensión de la realidad educativa y transformarla de acuerdo con la modalidad técnica y práctica que la caracteriza (Colmenares y Piñero, 2008). Se contó con la participación de 20 alumnos inscritos en la asignatura Cálculo Diferencial e Integral de 2. ${ }^{\circ}$ semestre de la licenciatura en Biotecnología de la Universidad Michoacana de San Nicolás de Hidalgo. Los participantes tienen una edad de entre 17 a 19 años. Se siguió la práctica "trae tu propio dispositivo" (BYOD, por sus siglas en inglés): los alumnos trabajaron con su smartphone y, desde ahí y a través de una conexión a Internet, programaron y accedieron a la calculadora gráfica Desmos para desarrollar la actividad. Después de experimentar con la app, se recogieron los datos a través de Kahoot!, plataforma que permite, a manera de juego, realizar un quiz y verificar las respuestas de manera instantánea; en este momento el uso de la app Desmos no se permitía. Durante toda 


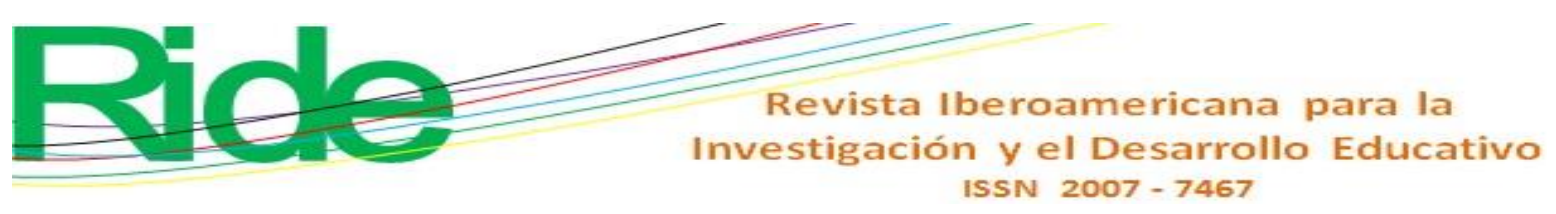

Es conveniente graficar en Desmos el renglón número ocho para hacer la comparación de los valores antes emitidos y permitir comparar la función derivada.

Asimismo, se recomienda inhabilitar el renglón cinco y ocho, como queda patente en la figura 2.

Figura 2. Visualización de la derivada en un punto con Desmos

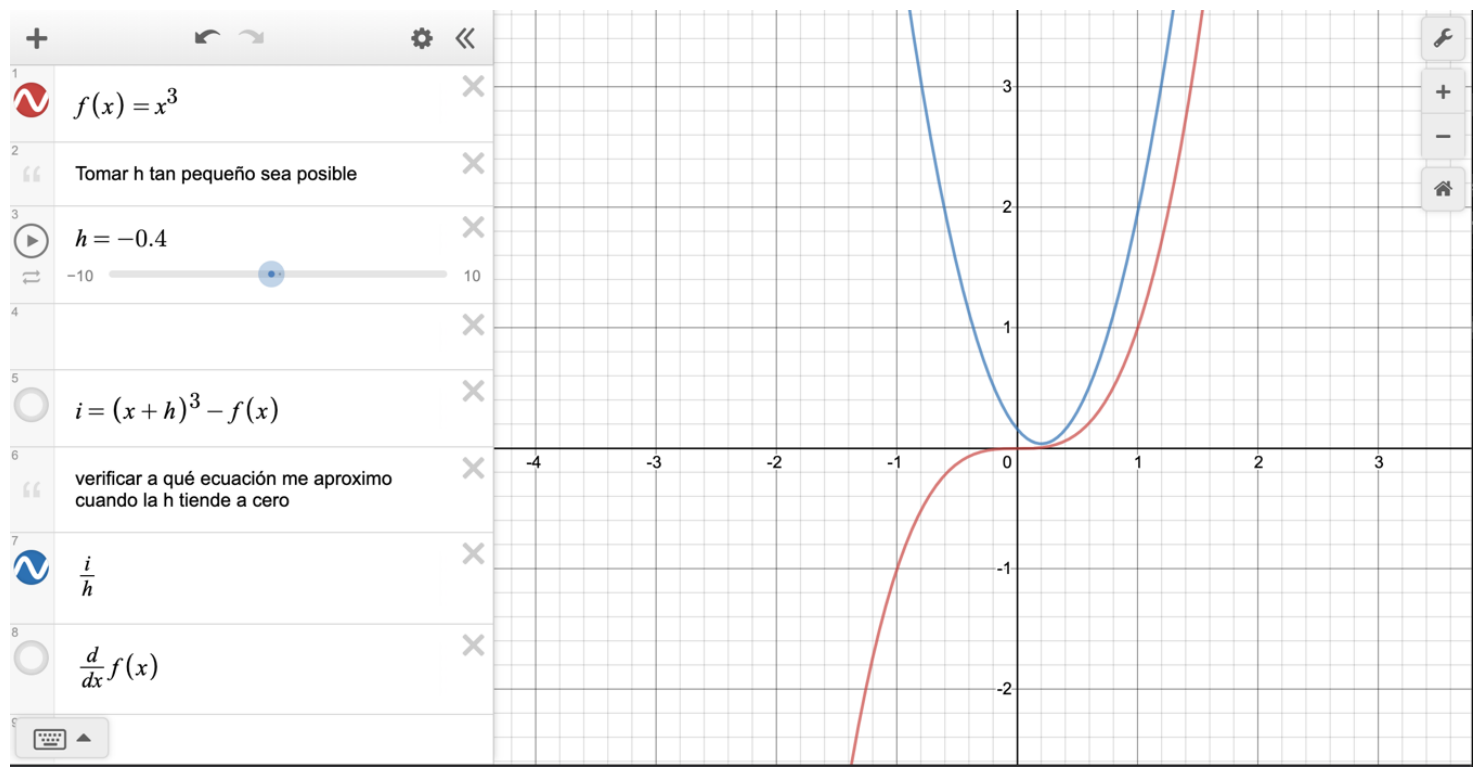

Fuente: Elaboración propia

Una vez realizado lo anterior, solo basta mover el deslizador de $h$ lo más cercano a cero, pues se busca que $\Delta x \rightarrow 0$, y después ejecutar la animación. 


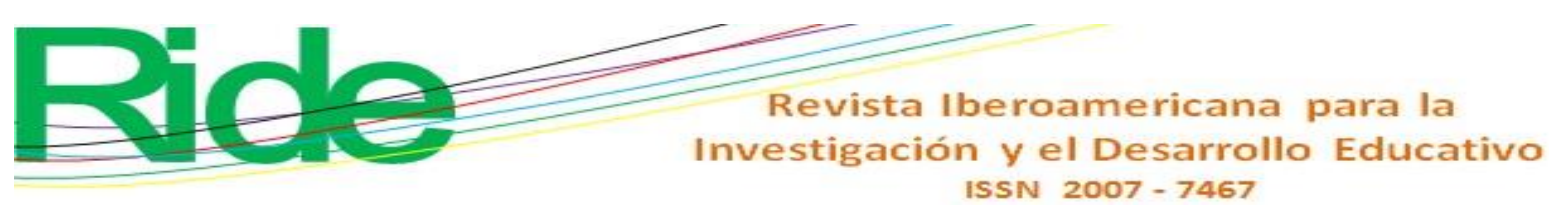

Figura 3. La derivada en un punto versus el límite de la razón de los incrementos cuando la variable dependiente tiende a cero mediante Desmos

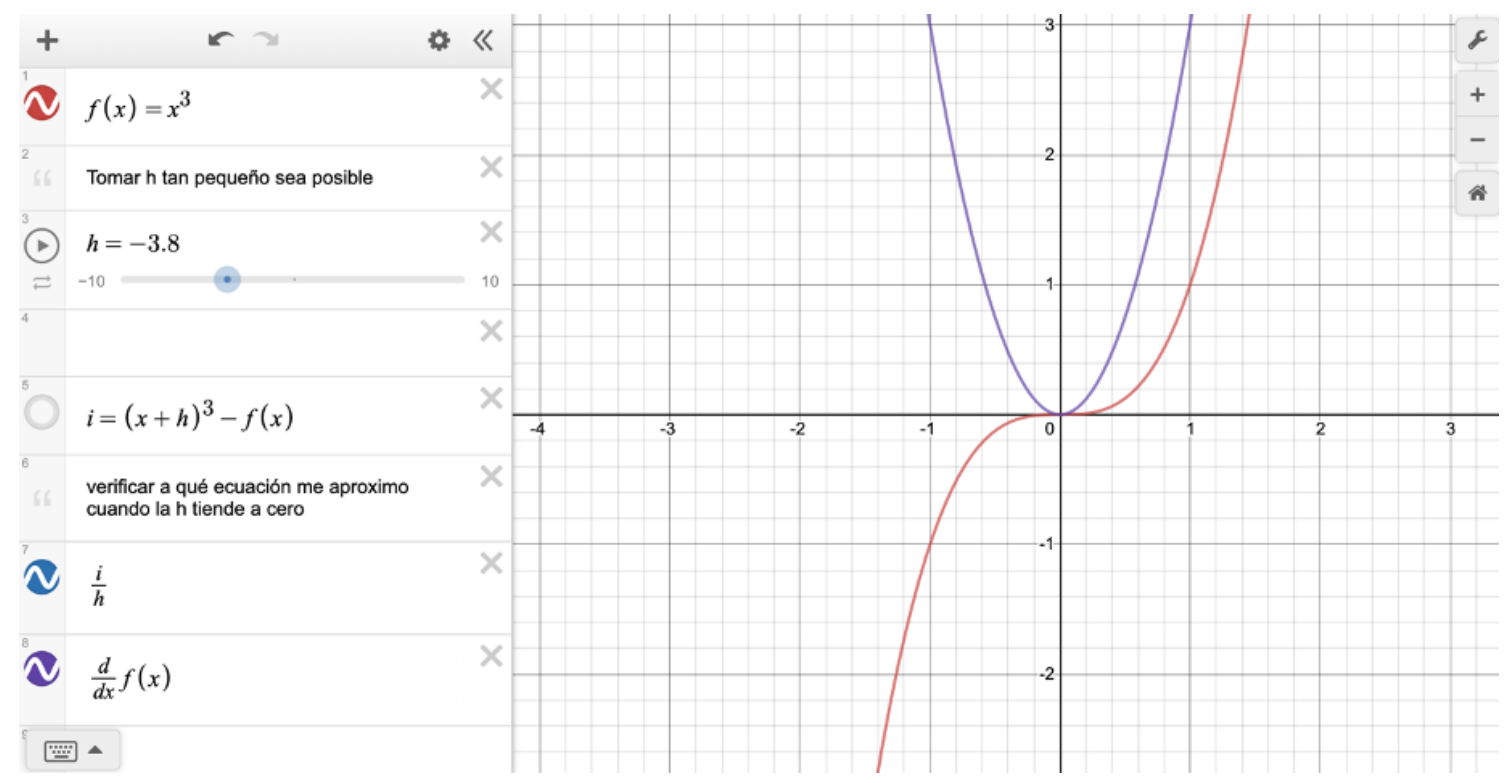

Fuente: Elaboración propia

El docente cuestiona a los alumnos con lo siguiente:

1) ¿Qué función esperan obtener al acercarse $h$ a cero?

2) ¿Qué función se obtiene cuando $h=0$ ?

3) ¿Qué es el límite de una función?

4) ¿Qué es la derivada de una función?

5) ¿Toda función es derivable?

\section{Resultados}

Después de la experimentación con la plataforma Desmos, se procede a realizar los cuestionamientos anteriormente señalados. Los resultados fueron capturados a través de cinco quizzes en la plataforma de Kahoot!, con un lapso de respuesta de hasta 60 segundos, excepto para el Quiz 5 (Q5), que fue hasta de 90 segundos por la complejidad que representa. Si el alumno contestaba fuera de ese intervalo de tiempo, la respuesta no se consideraba ni para puntaje del quiz ni para la estadística que se involucra en este trabajo.

Los resultados encontrados se observan en la figura 4 y en la tabla 1. 


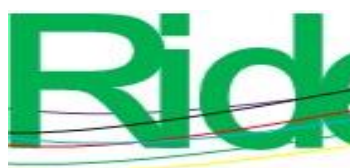

Revista Iberoamericana para la Investigación y el Desarrollo Educativo ISSN 2007 - 7467

Figura 4. Matriz de resultados obtenidos de la plataforma Kahoot!

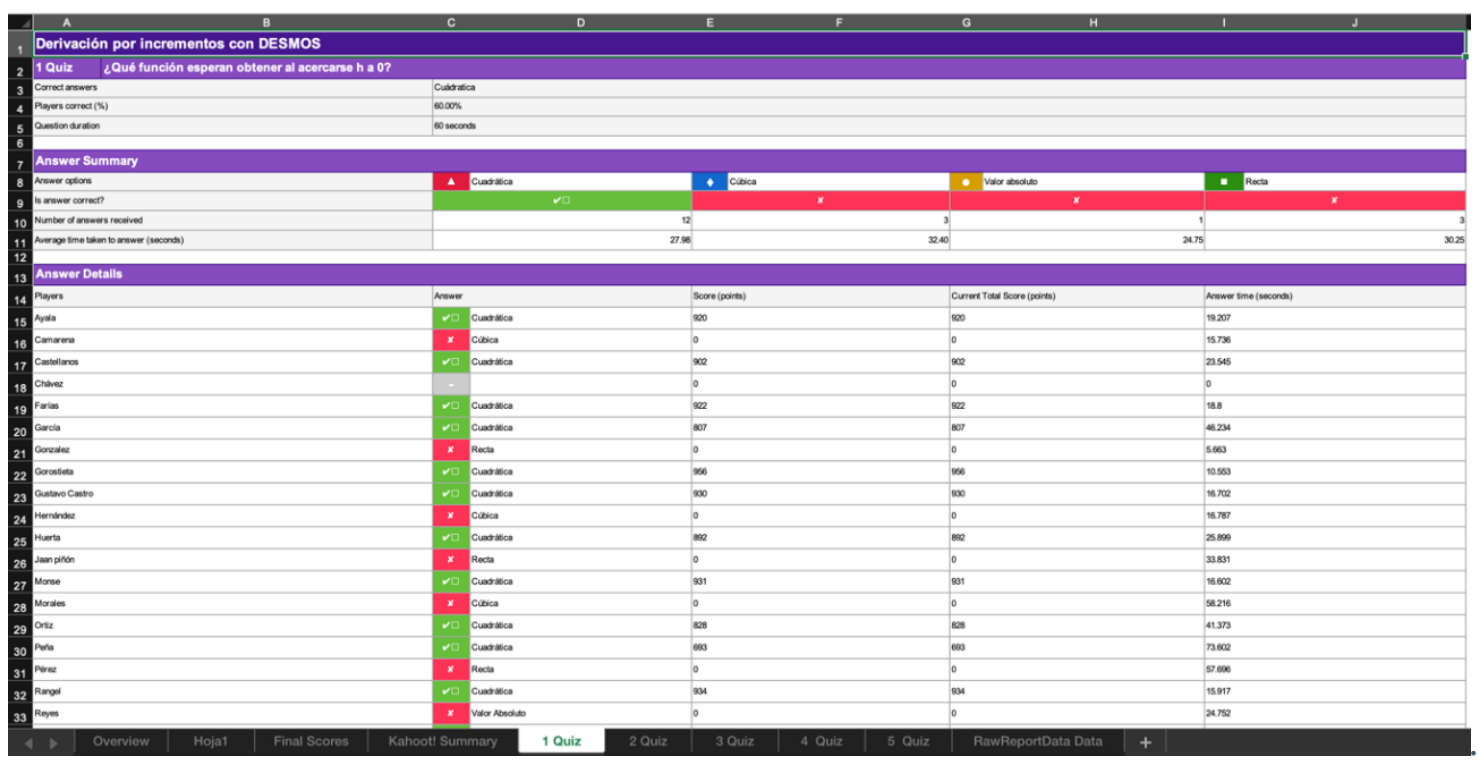

Fuente: Elaboración propia

Tabla 1. Distribución de respuestas

\begin{tabular}{|l|l|r|r|}
\hline & Quiz & Correctas & Incorrectas \\
\hline Válido & Q1 & $\mathbf{1 2}$ & $\mathbf{7}$ \\
\hline & Q2 & $\mathbf{9}$ & $\mathbf{1 1}$ \\
\hline & Q3 & $\mathbf{7}$ & $\mathbf{1 3}$ \\
\hline & Q4 & $\mathbf{1 9}$ & $\mathbf{1}$ \\
\hline & Q5 & $\mathbf{1}$ & $\mathbf{1 8}$ \\
\hline & Total & & $\mathbf{9 8}$ \\
\hline
\end{tabular}

Fuente: Elaboración propia

Dada la complejidad del Q5, se omite del análisis en las siguientes figuras, pues requiere una interpretación particular de sus resultados; además, no pertenece al objetivo del presente trabajo; resultaba inquietante, sin embargo, conocer hasta dónde podía llegar un análisis matemático. 

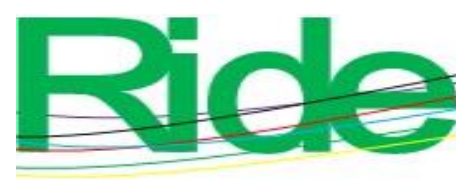

Revista Iberoamericana para la Investigación y el Desarrollo Educativo ISSN $2007-7467$

Figura 5. Desviación estándar de la distribución de respuestas por número de quiz

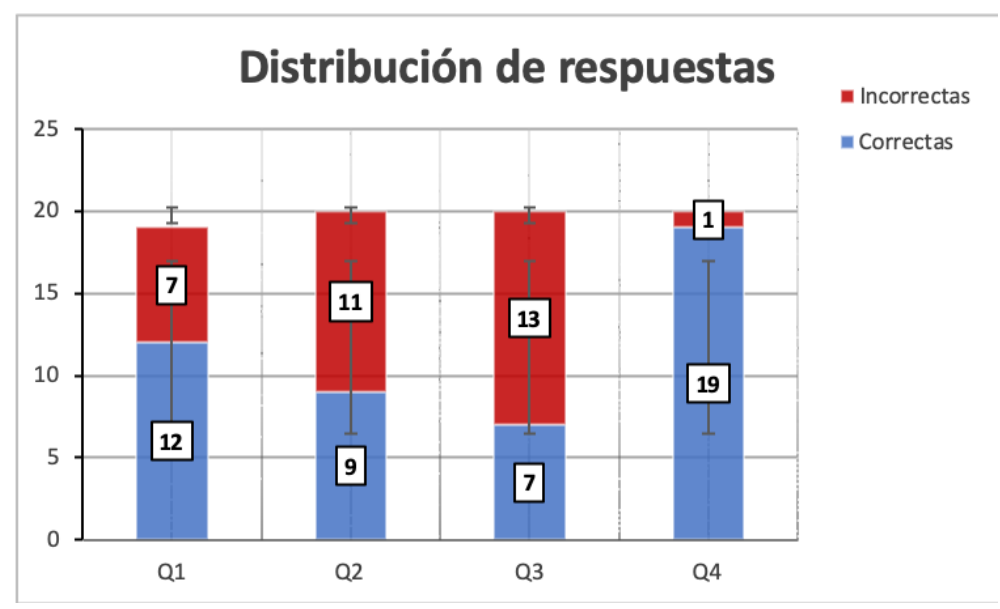

Fuente: Elaboración propia

Figura 6. Porcentaje de respuestas incorrectas por número de quiz

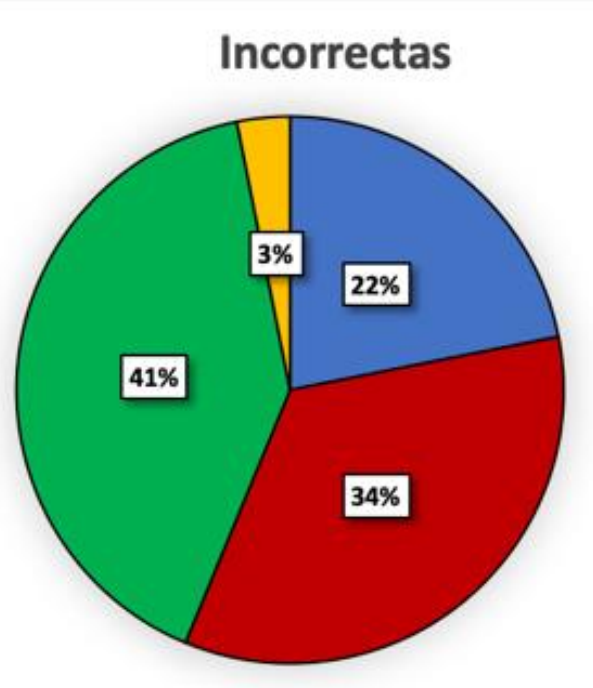

ロQ1

घQ2

वQ3

口Q4

Fuente: Elaboración propia 


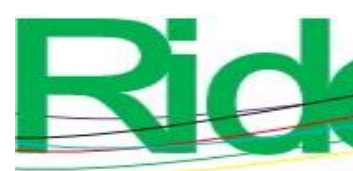

Revista Iberoamericana para la
Investigación y el Desarrollo Educativo
ISSN $2007-7467$

Figura 7. Porcentaje de respuestas correctas por número de quiz

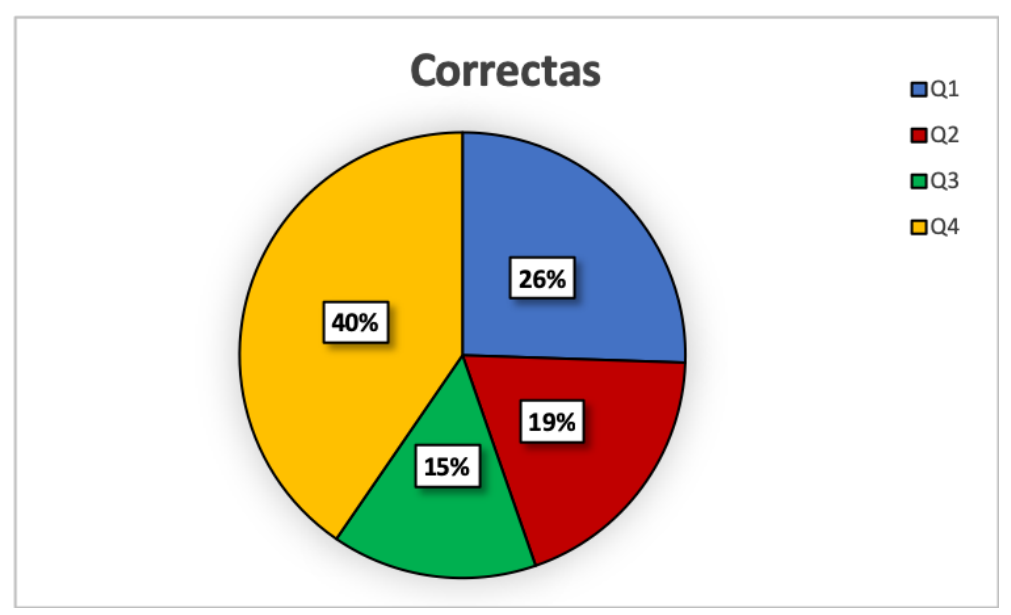

Fuente: Elaboración propia

Figura 6. Distribución de porcentajes totales de respuestas

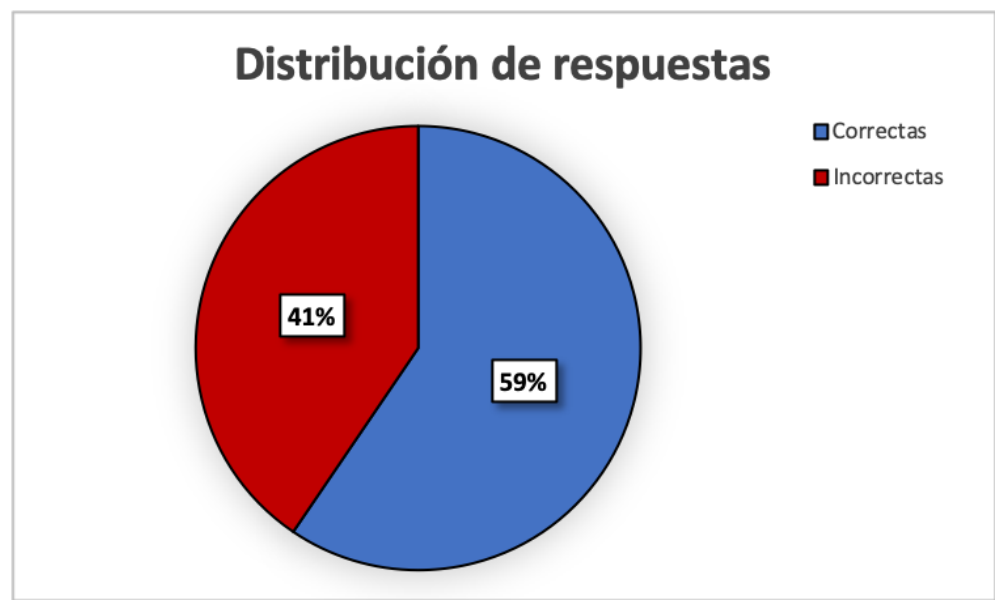

Fuente: Elaboración propia

\section{Discusión}

Ha quedado en evidencia que el uso de Desmos en el aula favorece a la comprensión del concepto de derivada de una función (Q4). Sin embargo, existen áreas de oportunidad.

Durante las observaciones que se hicieron en el aula al momento de la intervención con la plataforma, los alumnos mostraron confusión para realizar la programación. Pues el crear un deslizador lo asumían como punto; otros definían la función como $y$, donde matemáticamente es correcto pero en el algoritmo no es conveniente escribir la función como tal, sino definirla como una función que depende de una variable $x$. Al momento de terminar la programación en la plataforma y de ejecutar la animación, la actitud no fue de empatía ni 


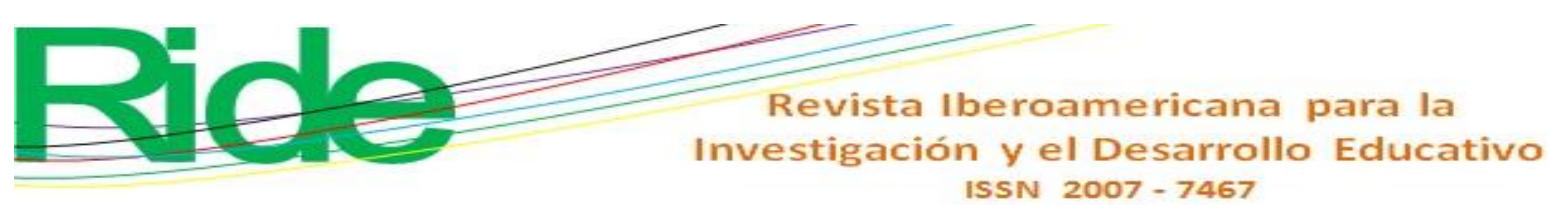

entusiasmo. Además, no se manifestó el reflejo de un aprendizaje, sino más bien la obediencia de seguir los pasos que el profesor les indicaba para realizar la tarea.

También quedó en evidencia que aún no logran comprender la definición de límite (Q3), de forma análoga a los suscitado en Sierra et al. (2000), pues, a pesar de haber sido tratada en una unidad temática previa, los alumnos no pudieron relacionarla: persiste la idea de que el límite se basa en una sustitución de la variable dependiente (matemáticamente hablando y no metodológicamente), sin importar que es imposible obtener su resultado. Por el contrario, la identificación de funciones (Q1) se vio muy favorecida al momento de graficar, pues lograron identificar, no de la manera exacta, sino más bien intuitiva, la función que se expresa matemáticamente.

El último quiz (Q5) tenía un grado de dificultad muy superior a los límites del objetivo en esta investigación trazado, pues para responderlo se requerían conocimientos superiores y de una asignatura avanzada, como lo es el análisis matemático; sin embargo, el desafiar a los alumnos puede resultar interesante, aunque es notorio que no respondieron de manera acertada, pero se crea el antecedente de que no siempre es posible que una función sea diferenciable en todo punto. Si bien un solo alumno acertó en la respuesta, no logró justificarla.

\section{Limitaciones}

\section{Tamaño de la muestra}

Las pruebas estadísticas normalmente requieren un tamaño de muestra más grande para asegurar una distribución representativa de la población y ser consideradas representativas de los grupos de personas estudiados. Aunque el tamaño de la muestra es menos relevante en la investigación cualitativa, es imperativo señalar que cuando hablamos de un proceso de enseñanza, y más en matemáticas, no debe abordarse ante un grupo masivo, a pesar de que algunos grupos en la realidad así estén estructurados, como en algunas escuelas o universidades. Por lo que si es un grupo más allá de 20 alumnos, la tecnología en el aula no es recomendada. 


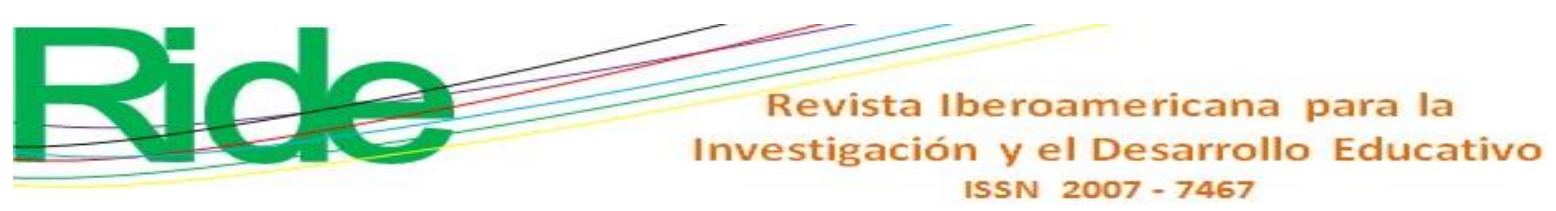

Infraestructura

Los alumnos deben de contar con un smartphone relativamente nuevo, donde se pueda descargar y actualizar la plataforma Desmos, así como contar con el uso de datos móviles o una conexión inalámbrica.

\section{Conocimiento de la plataforma}

El docente debe tener las habilidades y conocimientos de sistemas operativos comunes de smartphones para auxiliar en la ejecución y distribución de permisos adecuados para su correcta instalación y manejo de la plataforma Desmos.

\section{Conclusiones}

Se concluye de manera general que Desmos es un auxiliar de utilidad para la tarea de esbozo de una función, pues simplifica el cómputo y el trazado de funciones, y señala los puntos discontinuos que operacionalmente pudieran pasarse por alto. Pero la habilidad y la detección de los puntos problema, así como la interpretación de los resultados, sigue siendo terreno humano, es decir, no hay comprensión si no hay un desarrollo cerebral, un trabajo que rete a ello, y es aquí donde las habilidades individuales se ven exteriorizadas. El uso de la tecnología en el aula permite modelar ciertas realidades y comprender definiciones que ciertamente pueden ser complicadas de entender, pero el uso de la tecnología en matemáticas no es una forma válida de demostrar teoremas. A pesar de que es posible encontrar la derivada de una función a través de incrementos, no es conveniente recurrir a ello como único procedimiento. Pues, a pesar de ser un método holístico que incluye un límite, el factor tiempo puede jugar un papel ponderante, especialmente si es una función que necesita mucha experiencia algebraica o trigonométrica para ser encontrada de manera sintetizada. Por lo que se recomienda que se dé el paso al cálculo de la derivada por fórmulas. 


\section{Referencias}

Almarshedi, A., Wanick, V., Wills, G. B. and Ranchhod, A. (2017). Gamification and Behaviour. In Stieglitz, S., Lattemann, C., Robra-Bissantz, S. Zarnekow, R. and Brockmann, T. (eds.), Gamification. Using Game Elements y Serious Contexts (pp. 19-29). Switzerland: Springer. Retrieved from https://doi.org/10.1007/978-3-31945557-0.

Arrieta, J. y Díaz, L. (2015). Una perspectiva de la modelación desde la socioepistemología. Revista Latinoamericana de Investigación en Matemática Educativa, 18(1), 19-48. Recuperado de https://doi.org/10.12802/relime.13.1811.

Colmenares, A. M. y Piñero, M. L. (2008). La investigación acción. Una herramienta metodológica heurística para la comprensión y transformación de realidades y prácticas socio-educativas. Laurus, 14(27), 96-114.

Cottrill, J., Dubinsky, E., Nichols, D., Schwingendorf, K., Thomas, K. and Vidakovic, D. (1996). Understanding the limit concept: Beginning with a coordinated process scheme. Journal of Mathematical Behavior, 15(2), 167-193. Retrieved from https://doi.org/10.1016/S0732-3123(96)90015-2.

Ferrini-Mundy, J. and Gaudard, M. (1992). Secondary School Calculus: Preparation or Pitfall in the Study of College Calculus? Journal for Research in Mathematics Education, 23(1), 56-71. Retrieved from https://doi.org/10.2307/749164.

Ferrini-Mundy, J. and Graham, K. (1994). Research in Calculus Learning: Understanding of Limits, Derivatives, and Integrals. In Dubinsky, E. and Kaput, J. (eds.), Research issues in undergraduate mathematics learning (pp. 19-26). Washington, United States: Mathematical Association of America.

Habre, S. and Abboud, M. (2006). Students' conceptual understanding of a function and its derivative in an experimental calculus course. Journal of Mathematical Behavior, 21(1), 57-72. Retrieved from https://doi.org/10.1016/j.jmathb.2005.11.004.

Martínez, A., Cobos, J. C. y Torres, E. (2015). Matematización y modelización: experiencias y saberes. Una propuesta de aula. Espiral. Revista de Docencia e Investigación, 5(2), 9-22.

Recuperado de http://revistas.ustabuca.edu.co/index.php/ESPIRAL/article/view/1437/1135. 


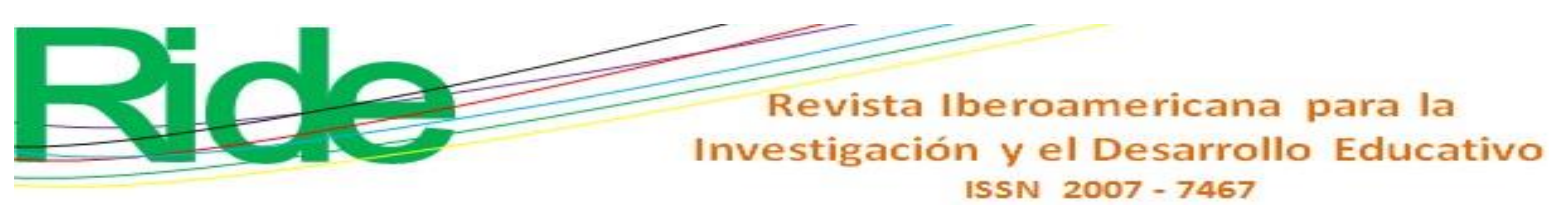

Martínez, J., López, R., Gras, A. y Torregrosa. (2002). La diferencial no es un incremento infinitesimal: evolución del concepto de diferencial y su clarificación en la enseñanza de la física. Enseñanza de las Ciencias: Revista de Investigación y Experiencias Didácticas, 20(2), 271-284.

Montijo, E. (2017). The Effects of Desmos and TI-83 Plus Graphing Calculators on the Problem-Solving Confidence of Middle and High School Mathematics Students. (doctoral dissertation). Liberty University, Lynchburg.

Rojas, E. R. (2015). Secuencias didácticas para la enseñanza del concepto de límite en el cálculo. Revista Internacional de Aprendizaje en Ciencia, Matemáticas y Tecnoloǵra, 2(2), 63-76.

Rojas, E. R. (2018). Matematización: una estrategia de enseñanza para mejorar el aprendizaje del cálculo. RIDE Revista Iberoamericana para la Investigación y el Desarrollo Educativo, 9(17), 277-294. Recuperado de https://doi.org/10.23913/ride.v9i17.381.

Rojas, E. R. (2019). Diseño de estrategia de apertura para la interpretación gráfica-analítica a través de Desmos como preparación para el aprendizaje del cálculo diferencial. RIDE Revista Iberoamericana para la Investigación y el Desarrollo Educativo, 10(19). Recuperado de https://doi.org/10.23913/ride.v10i19.493.

Sierra, M., González, M. y López, C. (2000). Concepciones de los alumnos de bachillerato y curso de orientación universitaria sobre límite funcional y continuidad. RELIME. Revista Latinoamericana de Investigación en Matemática Educativa, 3(1), 71-86.

Thomas, R. (2015). A graphing approach to algebra using Desmos. Paper presented at the 27th International Conference on Technology in Collegiate Mathematics. Las Vegas, March 12-15, 2015.

White, P. and Mitchelmore, M. (1996). Conceptual knowledge in introductory calculus. Journal for Research in Mathematics Education, 21(1), 79-95. Retrieved from https://doi.org/10.2307/749199. 